\title{
Los recursos fitogenéticos de piña (Ananas comosus var. comosus (L.) Merr.) en Cuba
}

\section{The phytogenetic resources of pineapple (Ananas comosus var. Comosus (L.) Merr.) in Cuba}

(iD) Daymara Rodríguez Alfonso ${ }^{1} \square$, (iD Miriam Isidrón Pérez ${ }^{1} y$ (iD Eduardo Menéndez Álvarez²

'Universidad Agraria de La Habana. Mayabeque, Cuba

Universidad Le Cordon Bleu. Lima, Perú

\begin{tabular}{llll}
\hline Recibido: $15 / 11 / 2019$ & Revisado: 10/01/2020 & Aceptado: 21/02/2020 & Publicado: 18/06/2020
\end{tabular}

\section{RESUMEN}

Los recursos fitogenéticos para la alimentación y la agricultura constituyen la base biológica de la seguridad alimentaria mundial y el sustento de la población mundial. Estos recursos son la materia prima más importante de los fitomejoradores y el aporte más imprescindible para los agricultores. La conservación in situ se ve cada día más afectada, es por ello por lo que se desarrollan actividades de capacitación para sensiblizar a los pobladores locales para salvaguardar la diversidad. En los últimos años se ha incrementado las alternativas de conservación ex situ, sobretodo las de largo plazo y la caracterización molecular e identificación de las variedades con técnicas de marcadores moleculares en las que se ha utilizado desde isoenzimas hasta secuenciamiento del genoma. El germoplasma de piña se mantiene fundamentalmente en tres grandes colecciones ubicadas en Brazil, Martinica y Hawaii. En Cuba la base genética de la especie no es amplia solo están representados los grupos horticulturales Española, Cayena y Pernambuco. La variedad predominante es 'Española roja' y las menos cultivados 'Piña blanca' y algunas Cayenas, por lo que puede peligrar su perpetuidad. Las mayores áreas dedicadas a la siembra de la piña en el país pertenecen al sector estatal pero la mayor diversidad la atesoran los campesinos.

Palabras clave: Variedades, conservación in situ, conservación ex situ, marcadores moleculares.

\begin{abstract}
The phytogenetic resources for food and agriculture constitute the biological basis of the world's food safety and the sustenance of world population. These resources are the most important raw material of the phyto-enhancers and the most indispensable contribution for farmers. Conservation in situ is being increasingly affected, and it is for this reason that training activities are carried out to sensitize the local population to safeguard diversity. In the past years the ex situ conservation alternatives have increased, particularly, the long-term ones and the molecular characterization and identification of the varieties with molecular markers techniques in which iso-enzymes up to genome sequencing have been used. The pineapple germplasm is mainly maintained in three great collections located in Brazil, Martinique and Hawaii. In Cuba, the genetic base of the species is not extensive,
\end{abstract}


and only the horticultural groups of Hispaniola, Cayenne and Pernambuco are represented. The predominant variety is the 'Red Spanish', while the While Pineapple and some Cayenne pineapples are the least grown, so their perpetuity could be endangered. The largest areas for the growing of pineapples in the country belong to the state, but the greatest diversity is kept by the farmers.

Keywords: Varieties, conservation in situ, conservation ex situ, molecular markers.

\section{INTRODUCCIÓN}

El hombre siempre ha utilizado los diferentes recursos disponibles en su entorno y entre ellos se incluyen las especies vegetales. De este modo, ha domesticado y adaptado un gran número de dichas especies con diferentes fines, dando lugar a numerosas variedades locales que constituyen una diversidad genética particular conocida como recursos fitogenéticos (Cubero, 2013).

El Tratado Internacional sobre Recursos Fitogenéticos para la Alimentación y la Agricultura (TIRFAA) los define como "cualquier material genético de origen vegetal de valor real o potencial para la alimentación y la agricultura" (FAO, 1998). Bajo esta definición, se incluyen las siguientes categorías de materiales: variedades locales o variedades comerciales de especies cultivadas; variedades en desuso, especies en desuso, especies silvestres o asilvestradas afines a las cultivadas, malas hierbas o arvenses y materiales obtenidos en trabajos de mejora genética (Esquinas-Alcázar, 1983; Cubero, 2013).

Los Recursos Fitogenéticos para la Alimentación y la Agricultura (RFAA) constituyen la seguridad para futuras necesidades que aún se desconocen, un gran número de variedades tradicionales genéticamente más heterogéneos, son reemplazados a diario por nuevos cultivares que tienden a ser más uniformes lo que aumenta su vulne- rabilidad. En los últimos 50 años, nuevas variedades uniformes de los principales cultivos han desplazado a cientos de variedades locales en grandes áreas de producción (INIFAT, 2001). Vavilov indicó en la década de 1920 y Harlan en la de 1930, una pérdida mundial de variedades tradicionales de los agricultores (Casas y Parra, 2007), que continúa incrementándose. Los recursos fitogenéticos en el mundo están desapareciendo a ritmo agigantado, esto sin dudas, representa serias implicaciones para el bienestar de la humanidad (Moore y Tymowski, 2008).

La familia Bromeliaceae se distribuye desde Chile y Argentina hasta el sureste de los Estados Unidos, pasan a través de Centroamérica y el Caribe, a excepción de la especie Pitcairnia feliciana (A. Chev. Harms y Mildbr.), que se encuentra en el oeste de África (Coppens y Leal, 2001). Su gran diseminación en el mundo es debido a la adaptabilidad de la familia a diferentes hábitats, desde el desierto semiárido hasta el bosque mesófilo de montaña y la selva alta perennifolia, así como a la altitud en las que son capaces de crecer, que varía desde el nivel del mar hasta 4500 m.s.n.m. (Bartholomew et al., 2002).

La piña es originaria de las regiones tropicales de América del Sur, en el centro y sureste de Brasil, noreste de Argentina y Paraguay. Aunque es oriunda de esta región, 
se cultiva en las zonas tropicales y subtropicales del muno debido a su adaptabilidad, tolerancia a la sequía y fácil manejo del material de propagación (Leal y Antoni, 1981; Coppens y Leal, 2003).

La domesticación de A. comosus comenzó en fecha incierta en algún sitio o en varios sitios simultáneamente. Bertoni (1919) planteó que surgió a partir de Ananas ananassoides (Baker) L. B. Sm. domesticada de los indios tupí-guaraníes. No obstante, León (2000) informó que no hay evidencia de que $A$. comosus pueda derivarse de otros congéneres de frutos comestibles como Ananas bracteatus (L.) Schult. y Schult. F. y Ananas erectifolius (L. B. Sm.), variedades cultivadas por los indios del Brasil y Paraguay utilizadas para obtener fibra de las hojas y no por sus frutos que tienen calidad muy inferior. Tampoco parece derivarse de especies morfológicamente afines como Ananas parguazensis (L. B. Sm.).

En A. comosus no se han descubierto aún poblaciones silvestres; los piñales que se han encontrado de manera espontánea en las selvas y sabanas del norte de América del Sur son restos de siembras abandonadas. La propagación vegetativa que caracteriza a esta especie permite a las plantas vivir por más de medio siglo sin la intervención del hombre (León, 2000; Bartholomew et al., 2002).

En el estado actual de conocimientos no se le puede asignar a la piña, ni un antecesor posible. En la domesticación debieron actuar varios factores de forma favorable, como la ausencia de semillas por la incompatibilidad presente, la facilidad de la propagación vegetativa y a menor escala, los mutantes con hojas sin espinas. Las mutaciones de yema fueron un material amplio para la selección de algunas variedades comerciales como 'Cayena', la cual descienden directamente de poblaciones primitivas (Coppens y Duval, 1995; León, 2000).

\section{Taxonomía}

Dentro de las 2921 especies agrupadas en 56 géneros que tiene la familia Bromeliaceae, la piña es la más conocida, pertenece al género Ananas y es cultivada como alimento. Esta familia se divide en tres subfamilias: Pitcarnioideae, Tillandsioideae y Bromelioideae, en las que se destacan especies como las de los géneros Tillandsia, Aechmea, Guzmania y Billbergia, entre otras, pero su relevancia se debe a su uso como plantas ornamentales de jardín o de interior (Coppens et al., 1997). La piña se clasifica según Cronquist (1981): División: Magnoliophyta, Clase: Liliopsida; Subclase: Zingiberidae; Orden: Bromeliales, Familia: Bromeliaceae, Género: Ananas, Especie: Ananas comosus.

Se han establecido varias clasificaciones taxonómicas de la especie, entre las más recientes se encuentran la de Coppens y Leal (2003) los que reconocen cinco variedades botánicas de $A$. comosus (Fig. 1): ananassoides, bracteatus, rectifolius, parguazensis y comosus. A la última pertenece la piña comestible, única con valor comercial en su fruto. En las restantes variedades se obtienen frutos conocidos como "piñuelas", que pueden ser o no consumidos, en dependencia de la región, pero tienen una elevada importancia como fuentes de genes para los programas de mejora genética, uso farmacéutico, obtención de fibra para la elaboración de sogas o papel, en la industria tanto con fines alimenticios como cosmética o por su valor ornamental (Souza et al., 2007; Leão et al., 2009; Coppens y Duval, 2009). 

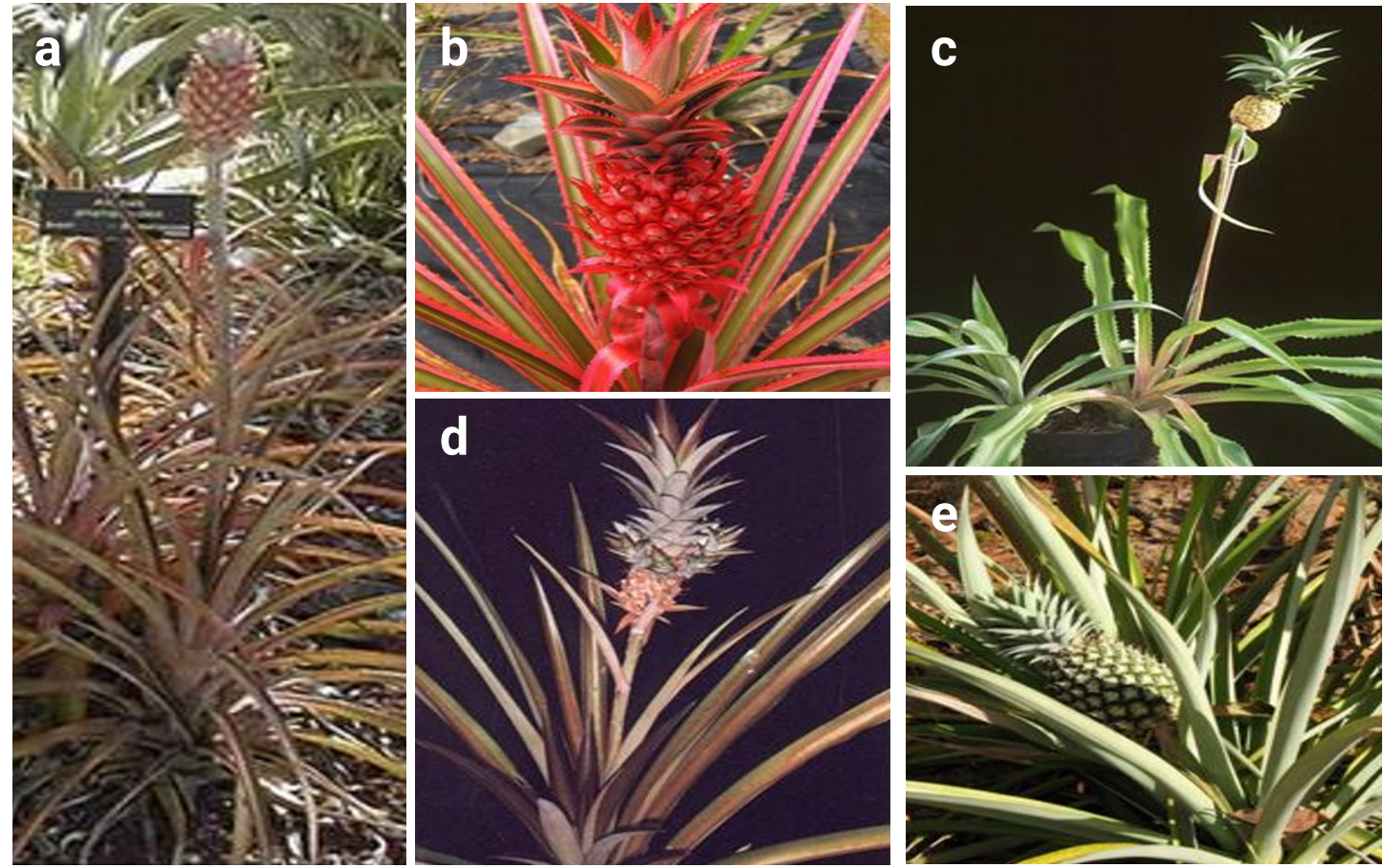

Figura 1. Variedades botánicas de A. comosus: a) ananassoides, b) bracteatus, c) parguazensis, d) rectifolius (sinonimia A. lucidus) tomado de (Berg y Berg, 2000) y e) comosus.

\section{Producción mundial}

Los países en desarrollo producen más del 95 $\%$ de los alimentos del mundo, donde el $75 \%$ de estos son frutos primarios y de ello, la piña ocupa un lugar importante en el comercio internacional (Reinhardt, 2007). Su producción a escala mundial en el año 2017 fue de 27402956 t, de esta el continente asiático ocupó más del 40 \% con 11873279 t, seguido de América con 9962986 t. Los cinco países que lideran la producción son Costa Rica con 3056445 t, que representa el $11 \%$ de la producción, Filipinas con 2671711 t, Brasil con 2253897 t, Tailandia con 2123177 t e India con 1861000 t (FAOSTAT, 2020).

\section{Características botánicas, anatómicas y citogenéticas}

La mayoría de las bromeliáceas son epífitas, pero la piña es una planta terrestre, con raíces superficiales. Es monocotiledónea, herbácea perenne y después de su fructificación continúa su crecimiento mediante una o más yemas axilares que dan origen a una nueva planta (Bartholomew et al., 2002).

La planta adulta puede alcanzar hasta 2,0 m de altura y $1,5 \mathrm{~m}$ de diámetro. El eje principal es un pseudotallo sobre el cual se disponen las hojas en forma de roseta, las cuales presentan debajo de la epidermis superior una capa de células que le confieren rigidez. Las hojas son sésiles, lanceoladas, con márgenes dotados o no de espinas, en dependencia de la variedad, ligeramente cóncavas, lo que permite conducir el agua de lluvia hacia la roseta y ser almacenada la humedad en el tejido acuífero, para dederla a la planta cuando hay sequía. Las hojas jóvenes adultas (conocidas como hoja D) son excelentes indicadores del estado fisiológico y nutricional de la planta (Py et al. 1987; Bartholomew et al., 2002).

La inflorescencia o espiga está formada por flores perfectas (hermafroditas), 
rosadas, que aparecen al final de un escapo en las axilas de las brácteas y son autoestériles. La polinización cruzada entre dos variedades es factible, dando lugar a semillas redondas, pequeñas y duras. Pero en la piña, lo normal es que no se produzca fecundación cruzada, sino que la flor mediante partenocarpia da origen a una pequeña baya y todas fusionadas forman el sincarpo (frutescencia) (Bartholomew et al., 2002; Coppens y Leal, 2003).

Se tiene conocimiento desde los primeros trabajos publicados por Collins y Kerns (1931) y Canpinpin y Rotor (1937) citados por Coppens y Duval (1995), que el número cromosómico de las especies del género Ananas es $2 n=2 x=50$, muy común entre las Bromeliáceas (Cotias de Oliveira et al., 2000), con la excepción de Pseudananas sagenarius (Arruda) Camargo que posee 100 cromosomas somáticos y es considerado un tetraploide $(2 \mathrm{n}=4 \mathrm{x}=100)$ (Collins, 1960). Los cromosomas son diminutos, casi esféricos y los valores de sus diámetros oscilan entre los 0,5 $\mu \mathrm{m}$ hasta 1,0 $\mu \mathrm{m}$ (Py et al., 1987).

Dentro de A. comosus pueden aparecer gametos gigantes no reducidos y producir triploides y tetraploides naturales (CoIlins, 1960). Entre las variedades triploides podemos encontrar "Ananas dos Indios" y "Cabezona" y entre los tetraploides 'James Queen' (Collins, 1960). En su mayoría, estos cultivares resultaron de la unión de gametos en los que no ocurrió reducción cromosómica durante la meiosis (Py et al., 1987).

\section{Clasificación de las variedades}

El origen de la mayoría de las variedades en piña puede atribuirse principalmente a las mutaciones somáticas, ya que no se produce autofecundación y ocasionalmente ocurre la polinización cruzada a través de la que se obtienen semillas viables. Por esta razón, los híbridos naturales son muy escasos (Bartholomew et al., 2002, Chan et al., 2003).

La clasificación de las variedades de piña cultivadas comercialmente ha sufrido varios cambios, desde la primera propuesta de Hume y Miller (1904) que la dividían en tres grupos (Cayena, Queen y Española) y en la que solo tenían en cuenta las similitudes morfológicas de las hojas, las espinas, el color de las flores, la forma y el tamaño del fruto. Posteriormente Py (1970) sugirió la adición de dos grupos más Pernambuco y Mordilona o Perolera; y finalmente Py et al. (1987) propone estos cinco grupos horticulturales para clasificar los cultivares de piña.

Esta agrupación fue criticada por Duval y Coppens (1993), bajo el argumento de que la clasificación justificada mediante razones prácticas presenta grandes limitaciones desde el punto de vista genético. La noción de grupo no tiene una definición constante, por ejemplo, las variedades de los grupos Pernambuco y Española, son definidos a través de criterios morfológicos, pero corresponden a varios genotipos diferentes, mientras que Cayena y Queen pueden ser comparados como "colecciones de clones" las que provienen de un mismo clon original y presentan una variación limitada, y el grupo Perolera es más variable y se define por poseer hojas con el carácter piping (margen involuto de las hojas).

\section{Características de los grupos horticulturales de piña}

Española: Sus plantas son de tamaño mediano, de hojas largas y estrechas, verde oscuro y con una banda central rojo cobriza, 
sus espinas se distribuyen de forma irregular o regular. El fruto es redondeado, naranja-rojo, de $1-2,5 \mathrm{~kg}$, con ojos muy definidos y grandes, al madurar es duro, lo que permite su fácil manipulación y transporte.La pulpa es amarilla pálida, fibrosa, tiene un contenido en azúcares medio y un bajo nivel de acidez (Py et al., 1987; Bartholomew et al., 2010). Es una planta tolerante a altas temperaturas y la sequía. Presenta varios hijos basales que son preferidos entre los agricultores para establecer nuevas plantaciones (Isidrón, 2002).

Pernambuco: Es muy conocida en Brasil; las plantas son de tamaño mediano, con hojas verdes, y espinas cortas, rectas muy unidas. El fruto es de forma piramidal, verde amarilloso de aproximadamente 1,0 a 2,0 kg y con ojos pequeños. La pulpa va desde amarillo pálido a blanca, de excelente sabor, suculenta, jugosa y con elevado contenido en azúcares totales. Se se utiliza como fruta fresca, pero no es apropiada para la comercialización debido a sus bajos rendimientos y la sensibilidad del fruto a la manipulación (Py et al., 1987; Bartholomew et al., 2010).

Cayena: Son plantas de porte mediano, con hojas más cortas y anchas que las del grupo Española, los bordes son lisos, a excepción de la porción apical y a veces en la base de las mismas presentan espinas cortas. El fruto es cilíndrico, naranja, de 1,5 y 2,5 $\mathrm{kg}$, ojos superficiales. La pulpa es amarilla, jugosa, de poca fibra, con un excelente sabor y elevado contenido en azúcares totales (Py et al., 1987; Bartholomew et al., 2010). Por las características antes expuestas y por la forma del fruto. Son muy apreciadas para el consumo fresco y la industria (Matos y Reinhardt, 2009).

Dentro de este grupo se destaca la 'MD-2' o 'Golden', un híbrido obtenido por Del Monte
Productos Frescos de Hawai. La planta tiene hojas con espinas del tipo Cayena, su fruto es de tamaño mediano a grande (1,3$2,5 \mathrm{~kg}$ ) y de un intenso amarillo-naranja. La pulpa es amarrilla, compacta, poco fibrosa, con bajo contenido en ácido ascórbico, un exquisito sabor y alto contenido en azúcares totales (Chan et al., 2003; Bartholomew et al., 2010). Al madurar la fruta se debe recolectar de inmediato, pues se sobremadura con rapidez en el campo (Isidrón, 2002). Debido a sus excelentes cualidades, agradable sabor, consistencia y contenido en azúcares totales, tiene una mejor aceptación entre los consumidores, por lo que ha desplazado a 'Cayena lisa' de mercados importantes como Estados Unidos y Europa (Del Angel y Rebolledo, 2007).

Queen: Está ampliamente distribuida, pero se suele cultivar más en el hemisferio Sur (África y Australia). Es una planta pequeña, de hojas verdes plateadas y espinosas. El fruto es cónico, pequeño (0,5-1kg), amarillo, con ojos profundos y con una larga vida útil comercial. La pulpa es amarilla dorada, crujiente, con alto contenido en azúcares totales, excelente sabor, poco jugosa y es menos fibrosa que Cayena (Chan et al., 2003; Bartholomew et al., 2010).

Perolera o Mordilona: Las variedades de este grupo predominan en el mercado brasileño. Son plantas grandes, de hojas cortas o medianas, verdes oscuros, con manchas rojizas, bordes lisos, con el carácter piping en las márgenes y un aguijón en la punta. Presentan frutos grandes de 1,5-3,5 kg, en forma de bloque cónico, verde amarillo y con ojos profundos. La pulpa es amarilla, jugosa, con exquisito aroma, con bajo contenido de azúcares totales y elevada concentración de ácido ascórbico (Leal, 1990; Bartholomew et al., 2002). Tiene alta resistencia a Fusarium 
sp. lo que ha permitido su inclusión en el desarrollo de programas de mejora para introducir este carácter en otras variedades (Cabral et al., 2003).

\section{Principales cultivares en Cuba}

El cultivo de la piña en Cuba se remonta hasta las postrimerías del siglo XIX (Isidrón et al., 2003). Se dedican más de 5000 ha a la plantación de esta fruta, las que se encuentran principalmente en la provincia Ciego de Ávila, al este del país (González, 2013).

La base genética de piña en el país es pobre, tradicionalmente se plantan los cultivares del grupo Española (Fig. 2a), que son los más distribuidos y los más utilizados por los agricultores, por esta razón 'Española roja' fue considera como la "reina" de los campos de Cuba. A pesar de que se caracterizan por tener espinas muy agresivas y frutos pequeños, presenta gran rusticidad y adaptabilidad, lo que explica sus aceptables rendimientos y su amplia distribución. Las variedades menos representadas son de los grupos horticulturales Pernambuco y Cayena (Fig. 2b y c). Estos, aunque poseen un fruto con mejor aceptación en el mercado por sus excelentes cualidades, requieren de mayores atenciones culturales y tienen menor rusticidad, lo que hace que los agricultores pierdan interés por cultivarlos (Isidrón et al., 2003, Rodríguez-Alfonso et al., 2017).
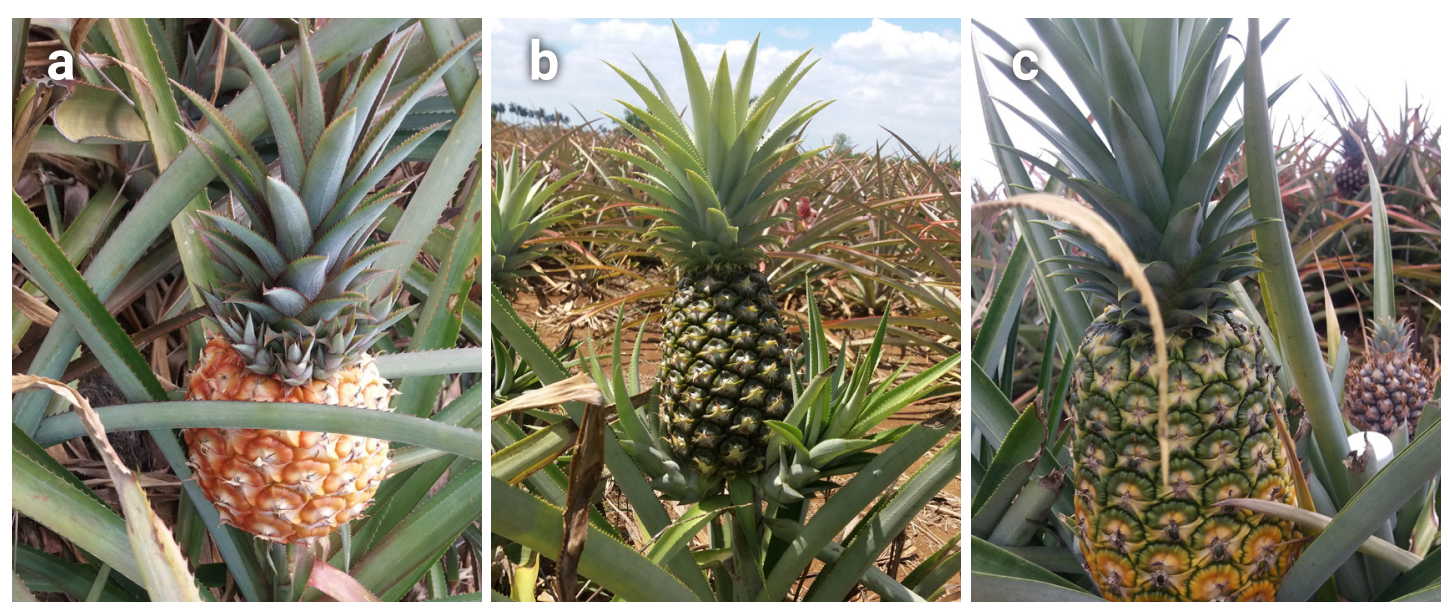

Figura 2. Grupos horticulturales de piña presentes en Cuba: a) Española, b) Pernambuco, c) Cayena

En el grupo Española también se encuentra la var. 'Cabezona', que es cultivado en la región oriental del país. En esta zona se siembra en bolsones de tierra entre rocas calizas. Su fruto, debido al gran tamaño y dulzor, goza de muy buena aceptación en el mercado (Isidrón, 2002).

En el grupo Cayena existen varias accesiones en los campos de producción como la var. "Cayena lisa serrana" que es un clon sin hijos basales y con buena adaptación a las condiciones climáticas del país. Este material se recolectó en áreas de campesinos, en la provincia Ciego de Ávila, donde se ha multiplicado espontáneamente por más de 40 años (Isidrón et al., 1999). La var. "Baron de Rothschild" está en producción y se diferencia de "Cayena lisa" por la presencia de un gran número de espinas pequeñas a lo largo de las hojas, (Isidrón et al., 2003; Rodríguez-Alfonso et al., 2017). 
En los últimos 10 años, se introdujo en la producción, el híbrido "MD-2", el que ha permitido la recuperación de la producción nacional en más de 3600 t, la mayor cifra de los últimos años, algo que no ocurría desde hacía más de medio siglo (González, 2013).

En el grupo Pernambuco se encuentra la var. "Piña blanca” o "Piña de Cuba”, de gran tradición en la población rural. Los campesinos cultivan un número pequeño de plantas para consumo familiar ya que su fruto tiene agradable sabor, dulzura y jugosidad (Isidrón et al., 2003), lo que la incluye en el grupo de alto riesgo de erosión como variedad (Rodríguez-Alfonso et al., 2017).

En el país también se plantan los híbridos CBCE-074 y CBCE-116, obtenidos por Benega et al. (1997) a partir del cruce entre "Cayena lisa serrana" como progenitor femenino (que presenta problemas de adaptación en condiciones de cultivo, pero muy buenas características en su fruto) y "Española roja pinareña" (que aporta gran rusticidad pero con una agresividad extrema de sus espinas). Estos se mantienen en pequeñas áreas.

\section{Conservación de los recursos fitogenéticos (RFG)}

La conservación de los RFG ha adquirido relevancia en las últimas décadas, no solo por la erosión sufren, lo que conlleva a la pérdida o disminución de la diversidad genética, sino también, por el valor potencial que poseen. Cuando se habla de preservación de germoplasma, hay que destacar que el objetivo es conservar con la mayor integridad posible la variabilidad genética de las poblaciones seleccionadas (Lobo y Medina, 2009).

Como consecuencia de la pérdida de la diversidad de los RFAA, se ha producido un esfuerzo de colecta y conservación, con estrategias para el mantenimiento de las poblaciones domesticadas (variedades de agricultor, variedades obsoletas, poblaciones de mejoramiento, variedades mejoradas y materiales especiales) y los silvestres relacionadas con las plantas cultivadas (INIFAT, 2001). Las dos formas de conservación existentes in situ y ex situ, no son excluyentes ya que entre ambas se puede desarrollar una estrategia complementaria.

La complementariedad de ambos tipos de conservación es especialmente importante en el caso de las especies relacionadas con las cultivadas (Fowler y Hodgkin, 2004). La planificación de estrategias óptimas para la conservación de plantas cultivadas y silvestres está afectada por factores biológicos y ambientales que deben considerarse desde el inicio.

\section{Conservación in situ}

La conservación in situ de los RFAA se refiere a su preservación en los ecosistemas y hábitats naturales y al mantenimiento y recuperación de poblaciones viables de especies en sus entornos naturales (Conferencia de las Naciones Unidas sobre el Medio Ambiente y el Desarrollo, 1992). Este es un término que toma relevancia en la década de los 80 del pasado siglo, como una importante estrategia de conservación de la diversidad.

Gran parte de la agrobiodiversidad remanente in situ se encuentra en las fincas de semisubsistencia de los países más pobres y en los "jardines caseros" de las naciones industrializadas (Brookfield et al., 2002; IPGRI, 2003). El apoyo al mantenimiento in situ de los RFG contribuye de manera decisiva a que las comunidades locales se sientan con capacidad y más seguras en término de alimentos e ingresos (Engels y Visser, 2007). 
La sostenibilidad a largo plazo de los RFG de piña depende del mantenimiento in situ de la diversidad genética de la especie. La principal fuente de germoplasma en el mundo se localiza en la región de origen de las cuencas del Amazonas y del Orinoco. La pérdida de identidad cultural y la implementación de nuevas tecnologías en el mundo han puesto en riesgos una parte considerable de los RFG de la especie (Duval et al., 1996).

En Cuba, Isidrón et al. (2003) y RodríguezAlfonso et al. (2017) en prospecciones realizadas, detectaron pérdida de la diversidad en la piña. No obstante, el mantenimiento in situ de los RFG ha permitido la reintroducción de accesiones al banco de germoplasma que se estaban dañadas por diversas causas bióticas y abióticas y el incremento de la diversidad en la colección.

\section{Conservación ex situ}

La conservación ex situ de los RFAA es el mantenimiento de componentes de la diversidad fuera de sus hábitats naturales, esta se realiza en los bancos de germoplasma, jardines botánicos y en programas de mejoramiento (Conferencia de las Naciones Unidas sobre el Medio Ambiente y el Desarrollo, 1992). Constituye el método principal de conservación para la agricultura a escala mundial.

En el mundo se han desarrollado diversas alternativas de conservación ex situ para piña como la conservación in vitro (mediano plazo) y la crioconservación (largo plazo) (GonzálezArnao y Engelmann, 2013). En Cuba, los protocolos establecidos para estas técnicas se han ajustado por García et al. (2007); Martínez-Montero et al. (2013) para lograr el mantenimiento de la colección nacional de piña.

Otra alternativa de conservación es el banco de germoplasma: lugar donde se guardan las partes reproductoras de las plantas ya sean: semillas, esporas, polen, bulbos, estaquillas $u$ otros propágulos vegetales de forma claramente identificables y fácilmente accesibles. Su importancia está directamente relacionada con la conservación de los RFG que son de vital necesidad en la agricultura moderna, debido a los riesgos de pérdida y de reducción de individuos debido a la destrucción del hábitat, desastres naturales o negligencias humanas que se han producido en las últimas décadas (Lobo y Medina, 2009).

Los recursos genéticos de piña en el mundo están conservados en bancos de germoplamas en diferentes países, el mayor se encuentra en el CNPMF perteneciente a la EMBRAPA en Cruz das Almas, Brasil con más de 800 accesiones; le sigue el del CIRAD- FLHOR de Martinica con más de 450 accesiones y el del USDA de Hawai con más de 130 accesiones. Aunque existen otras colecciones importantes, pero de menor tamaño como la de Venezuela, Malasia, Okinawa, México, Tailandia y Cuba (Coppens et al. 1997; Bartholomew et al., 2002, 2010).

\section{Caracterización del germoplasma de piña mediante marcadores morfológicos, bioquímicos y moleculares}

Históricamente, la caracterización e identificación de variedades se ha basado en el empleo de caracteres genéticos. Un marcador genético es un segmento de ADN con una ubicación física identificable en un cromosoma y cuya herencia se puede rastrear en una familia. Son utilizados a menudo como una forma indirecta de rastrear el patrón hereditario de uno o más genes (Cubero, 2013).

Los diversos tipos de marcadores genéticos estánagrupados en morfológicosymoleculares. Dentro de los moleculares, se encuentran las proteínas y los marcadores de ADN. También 
existen otros tipos de marcadores, pero en este caso asociados a características de la estructura y morfología de los cromosomas llamados marcadores citogenéticos, que tienen poco uso debido a la complejidad y dificultad en su medición (Azofeifa, 2006).

Los marcadores morfoagronómicos en piña se han utilizado para explorar la diversidad existente en las colecciones más importantes del mundo Brasil, Hawai y Martinica y en colecciones más pequeñas como la del Centro Nacional de Recursos Fitogenéticos de Venezuela (Bartholomew et al., 2002).

La aplicación de los distintos tipos de marcadores moleculares (RFLP, RAPD, ISSR y SSR) en piña, han tenido diferentes fines (Tabla 1). En su mayoría se han utilizado para esclarecer los problemas taxonómicos del género (DeWald et al., 1992; Sripaoraya et al., 2001; Ruas et al., 2001; Kato et al., 2004).Así como también para caracterizar las accesiones de los bancos de germoplasma: RFLP en la colección ex situ de Brasil (Duval et al., 2001), AFLP, RAPD e ISSR en 15 genotipos de la colección mexicana (Tapia et al., 2005), AFLP en genotipos de las colecciones de México (Yanes et al., 2012) y SSR en las colecciones de China y Tanzania (Feng et al., 2013; Makaranga et al., 2018). En Cuba, para determinar la diversidad genética del germoplasma se han utilizado los marcadores AFLP (Yanes et al., 2012), RAPD (Rodríguez et al., 2012) y SSR (Rodríguez et al., 2013).

\section{CONCLUSIONES}

La poca diversidad del germoplasma de piña en Cuba imposibilita abordar proyectos que contemplen programas de mejoras en este cultivo, por lo que se hace necesario ampliar ese fondo genético cuanto antes, incluyendo, además, variedades de los grupos Queen y Parolera.
El empleo de marcadores moleculares ha demostrado ser una herramienta eficaz en la determinación de la diversidad genética, las relaciones genéticas, la variabilidad, entre otros parámetros importantes de tener presente en las colecciones de piña.

\section{REFERENCIAS BIBLIOGRÁFICAS}

Azofeifa, A. (2006). Uso de Marcadores moleculares en plantas, aplicaciones en frutales del trópico. Agronomía Mesoamericana 17(2): 221-241.

Bartholomew, D., Paull, R. y Rohrbach, K. (2002). The pineapple, Botany, production and uses. University of Hawaii at Manoa, Honolulu, USA, 300pp.

Bartholomew, D., Coppens d'Eeckenbrugge G. y Ching-Cheng C. (2010). Pineapple. Hortscience 45(5): 740-742.

Benega, R., Cisneros, A., Isidrón, M., J. A., Martínez, J., Pérez G., Arias E. y Hidalgo M., (1997). Obtención y selección de híbridos promissórios de piña entre Cayena lisa serrana y Española roja. Cultivos Tropicales 18(3):72-75.

Berg, W. y Berg, D. (2000). Bromeliad Photo. Index - Florida Council of Bromeliad Societies. Disponible en: http://fcbs.org/pictures/ bergcage.htm (Consultado: 15 octubre de 2019).

Bertoni, M. (1919). Contribution à l'étude botanique des plantes cultivées. I. Essai d'une monographie du genre Ananas. Anal. Cient. Parag. (Serie II) 4: 250-322.

Brookfield, H., Padoch, C., Parsons, H. y Stocking, M. (2002). Cultivating Biodiversity: Understanding, Analysing and 
Using Agricultural Diversity. The United Nations University, London. ITDG Publications.

Cabral, J. R. S., Matos, A. P. y Junghans, D. T. (2003). Desenvolvimento de híbridos de abacaxi resistentes à fusariose. Cruz da Almas, BA, Embrapa-CNPMF, Comunicado Técnico 88: 4.

Canpinpin, J., Rotor, G. (1937). A cytological and morphogenetic study of some pineapple varieties and their mutants and hybrid delivatives. The Philippine Agriculturist 26(2): 139-158.

Carlier, J. D., Sousa, N. H., Espírito, T., Coppens d'Eeckenbrugge, G. y Leitão J. M. (2012). A genetic map of pineapple (Ananas comosus (L.) Merr.) including SCAR, CAPS, SSR and EST-SSR markers. Mol. Breed. 29: 245-260.

Casas, A. y Parra, F. (2007). Agrobiodiversidad, parientes silvestres y cultura. Revista de Agroecología. LEISA 23(2): 5-8.

Chan, Y. K., Coppens d'Eeckenbrugge, G. y Sanewski, G. (2003). Breeding and variety Improvement. En: Bartholomew, D.; Paull, R.; Rohrbach, K. (eds.). The Pineapple: Botany, Production and uses. CAB International, 33pp.

Charafi, J., El Meziane, A., Moukhli, A., Boulouha, B., El Modafar, C. y Khadari, B. (2008). Menara gardens: a Moroccan olive germplasm collection identified by a SSR locus-based genetic study. Genetic Resources and Crop Evolution 55(6): 893-900.

Collins, J. (1960). The pineapple: botany, cultivation and utilization. London, L. Hill; New York, 294pp.
Collins, J. y Kerns, K. (1931). Genetic studies of the pineapple. I. A preliminary report upon the chromosome number and meiosis in seven pineapple varieties (Ananas sativus L.) and in Bromelia pinguin L. J. Hered. 22: 139142.

Conferencia de las Naciones Unidas sobre el Medio Ambiente y el Desarrollo. (1992). Cumbre de la Tierra. Disponible en: http:// www.ecured.p.h.p./Cumbre_de_la_Tierra_de Rio_de_Janeiro (Consultado: octubre de 2019).

Coppens d'Eeckenbrugge, G. y Duval, M. F. (1995). Bases genéticas para definir una estrategia del mejoramiento de la piña. Rev. Fac. Agron. (Maracay) 21: 95-118.

Coppens d'Eeckenbrugge, G., Leal, F. y Duval, M. F. (1997). Germplasm Resources of Pineapple. Hortic. Rev. 21: 133-175.

Coppens d'Eeckenbrugge, G. y Leal, F. (2001). Pineapple. Disponible en: http:// www.ciat.cgiar.org/ipgri/fruit_from_americas/frutales/more\%20about\%20pineapple.htm5. (Consultado: 10 diciembre de 2019).

Coppens d’Eeckenbrugge, G. y Leal, F. (2003). Morphology, Anatomy and taxonomy. En: Bartholomew, D., Paull, R., Rohrbach, K. (eds.). The Pineapple: Botany, production and uses. CABI Publishing, Oxon, UK, 13-22.

Coppens d'Eeckenbrugge, G. y Duval, M. F. (2009). The domestications of pineapple: context and hypotheses. Pineapple News 16: 15-26.

Cotias de Oliveira, A., Assis, J., Bellintani, M., Andrade, J. y Guedes, M. (2000). Chromosome numbers in Bromeliaceae. Genet. Mol. Biol. 23: 173-248. 
Cronquist, A. (1981). An Integrated System of Classification of Flowering Plants. Columbia University Press.

Cubero, J. L. (2013). Introducción a la mejora Genética Vegetal. Ediciones Mundi Prensa, España, 569pp.

Del Angel, A. y Rebolledo, A. (2007). MD-2 pineapple in Mexico: introduction, evolution and perspectives. VI International Pineapple Symposium. Brazil, 62pp.

DeWald, M. G, Moore, G. A. y Sherman, W. B. (1992). Isoenzymes in Ananas (Pineapple): genetics and usefulness in taxonomy. J. Amer. Soc. Hort. Sci. 117: 491-496.

Duval, M. F. y Coppens d'Eeckenbrugge, G. (1993). Genetic variability in the genus Ananas. Acta Hort. 334: 27-37.

Duval, M. F., Coppens d'Eeckenbrugge, G., Ferreira, F., Cabral, J., Bianchetti, B. (1996). First results from joint EMBRAPA-CIRAD Ananas germplasm collecting in Brazil and French Guyana. Acta Hort. 160-172.

Duval, M. F., Noyer, J., Perrier, X., Coppens d'Eeckenbrugge, G. y Hamon, P. (2001). Molecular diversity in pineapple assessed by RFLP markers. Theor. Appl. Genet. 102: 83-90.

Engels, J. M. M. y Visser, L. (eds.). (2007). Guía para el manejo eficaz de un banco de germoplasma. Manuales para Bancos de Germoplasma. No.6. ISBN: 978-92-9043767-3. Bioversity International, Roma, Italia.

Esquinas-Alcázar, J. T. (1983). Los recursos fitogenéticos una inversión segura para el futuro. INIA, Madrid, 44pp.
FAO. (1998). The state of the World's Plant Genetic Resources for Food and Agriculture. Rome, FAO.

FAOSTAT. (2020). Food and Agriculture Organisation of the United Nations. Rome, Italy. Disponible en: http://www.fao.org. (Consultado: 11 enero 2020).

Feng, S., Tong $H_{\text {., }}$ Chen, Y., Wang, J., Chen, Y., Sun, G., He, J. y Wu, Y. (2013). Development of pineapple microsatellite markers and germplasm genetic diversity analysis. BioMed Research International http://dx.doi. org/10.1155/2013/317912.

Fowler, C. y Hodgkin, T. (2004). Plant genetic resources for food and agriculture: Assessing global availability. Ann. Rev. Environ. Res. 29: 143-79.

García, L., de Feria, M. y Acosta, K. (2007). Aspectos básicos de la conservación in vitro de germoplasma vegetal. Biotecnología Vegetal 7(2): $67-79$.

González, O. (2013). Otra reina busca el trono. Periódico Granma, La Habana, Cuba. Año 17 Núm. 128.

Hume, H. y Miller, H. (1904). Pineapple Culture II: Varieties. Florida. Agr. Exp. Sta. Bul. 70: 36-62.

González-Arnao, M. T. y Engelmann, F. (2013). Crioconservación de las plantas en América latina y el Caribe. San José, C.R.: IICA, 217 pp.

INIFAT (eds.). (2001). Lecciones avanzadas sobre conservación y manejo de recursos fitogenéticos. INIFAT. Ciudad de La Habana, Cuba, 328 pp. 
IPGRI. (2003). Home Gardens and the In situ Conservation of Plant Genetic Resources, Rome: International Plant Genetic Resources Institute. Disponible en: http:// www.ipgri.cgiar.org/system/page.asp?frame=publications/indexpub.htm. (Consultado: 20 marzo de 2010).

Isidrón, M. (2002). Algunas consideraciones técnicas acerca del establecimiento y atenciones al cultivo de la piña. Libro electrónico: Ed. Ciego de Ávila, Centro de Bioplantas, 41pp.

Isidrón, M., Benega, R., Cisneros, A., Arias, E., Lorenzo, J. C., Espinosa, P. y Borroto, C. (1999). Application of biotechnological and traditional methods in Cuban pineapple breeding program. Pineapples News 6: 17.

Isidrón, M., Rosales, Y., Pifferrer, A., Cisneros, A., Benega, R. y Carvajal, C. (2003). Caracterización del germoplasma de piña colectado en Cuba mediante prospección nacional: I. Localización, diversidad genética y situación actual. Revista Cultivos Tropicales 24(1): 65-71.

Kato, C., Nagai, C., Moore, P., Zee, F., Kim, M., Steiger, D. y Ming, R. (2004). Intra-specific DNA polymorphism in pineapple (Ananas comosus L. Merr.) assessed by AFLP markers. Genet. Res. Crop Evol. 51(8): 815- 825.

Leal, F. (1990). Complemento a la clave para la identificación de las variedades comerciales de piña [Ananas comosus (L.) Merr.] Rev. Fac. Agron. (Maracay) 16(1): 1-11.

Leal, F. y Antoni, M. G. (1981). Descripción y clave de las variedades de piña cultivadas en Venezuela. Rev. Fac. Agron. (Maracay), Alcance 29:51-79.
Leal, F., García, M. y Cabot, M. (1986). Prospección y recolección de Ananas y sus congéneres en Venezuela. Plant Genet. Resour. Newsl 66: 16-19.

Leão, A. L., Machado, I. S., Souza, S. F. y Soriano, L. (2009). Production of curaua fibers for industrial applications: characterization and micropropagation. Acta Hort. 822: 227-238.

León, J. (2000). Botánica de los cultivos tropicales. Tercera edición. IICA. San José, Costa Rica, 456 pp.

Lobo, M. y Medina, C. I. (2009). Conservación de recursos genéticos de la agrobiodiversidad como apoyo al desarrollo de sistemas de producción sostenibles. Revista Corpoica-Ciencia y Tecnología Agropecuaria $10(1)$ : 33-42.

Makaranga, A., Seth, M., Ndee, A., Mneney, E., Mbwambo, G., Lema, K., Godfrey, A., Mrema, L., Kachiwile, A., Mrema, E. y Msogoya, T. J. (2018). Diversity and genetic identity of pineapple [Ananas comosus (L.) Merr.] in Tanzania based on microsatellite markers. Revista African Journal of Biotechnology 17(26): 811-817. DOI: 10.5897/ AJB2018.16498.

Martínez-Montero, M. E., González-Arnao, M. T., Torres, M. A., García, L. y Fundora, Z. (2013). Desarrollo de la crioconservación de las plantas en Cuba. En: González-Arnao M.T., Engelmann F. Crioconservación de plantas en América Latina y el Caribe. San José, C.R.: IICA, 134-136.

Matos, A. y Reinhardt, D. (2009). Pineapple in Brazil: Characteristics, Research and Perspectives. Proc. VIth IS on Pineapple. Acta Hort. 822: 25-33. 
Moore, G. y Tymowski, W. (2008). Guía explicativa del Tratado Internacional sobre los Recursos Fitogenéticos para la Alimentación y la Agricultura. Seminario Internacional sobre la ejecución del Tratado Internacional sobre RFGAA. Cartagena de Indias, Colombia. UICN, Gland, Suiza, 223 pp.

Pérez, G., Yanes, E., Isidrón, M. y Lorenzo, J. C. (2009). Phenotypic and AFLP characterization of two new pineapple somaclones derived from in vitro culture. Plant. Cell. Tiss. Organ. Cult. 96: 113-116.

Py, C. (1970). La piña Tropical. Editorial Blume, Madrid, 568 pp.

Py, C., Lacoeuilhe, J. y Teisson, C. (1987). The pineapple: Cultivation and Uses. G.P. Maisonneuve et Larose, París, 568 pp.

Reinhardt, A. (2007). Industrial processing of pineapple-Trends and perspectives. VI International Pineapple Symposium, Brazil, 127 pp.

Rodríguez, D., Isidrón, M., Hormaza, J. I., Petit, S., Villar, P., Grajal-Martín, M. J. (2012). Genetic Characterization of the Cuban pineapple collection by RAPD. Revista Pineapple News 19: 24-25.

Rodríguez, D., Grajal-Martín, M. J., Isidrón, M., Petit, S., Hormaza, J. I. (2013). Polymorphic microsatellite markers in pineapple (Ananas comosus (L.) Merrill). Scientia Horticulturae 156: 127-130.

Rodríguez-Alfonso, D., Isidrón-Pérez, M., Alfonso-González, D., Grajal-Martín, M. J., Hormaza-Uroz, J. I, Herrera-Isidrón, L. (2017). Diversity of pineapple genetic resources in Cuba: threats and actions for minimizing losses. Rev. Fitotec. Mex. 40 (1): 93 - 101.
Souza, F. V. D., Cabral, J. R. S., Souza, E. H., Santos, O. N., Santos-Serejo, J. A., Ferreira, F. R. (2007). Caracterização morfológica de abacaxizeiros ornamentais. Magistra 19: 319-325.

Sripaoraya, S., Blackhall, N., Marchant, R., Power, J., Lowe, K., Davey, M. (2001). Relationships in pineapple by random amplified polymorphic DNA (RAPD) analysis. Plant Breed. 120(3): 265-267.

Tapia, E., Gutiérrez, A., Guillén, H., Gutiérrez, M. (2005). Caracterización genética de materiales de piña (Ananas spp.) mediante RAPD e ISSR. Revista Fitotecnia Mexicana. Chapingo, México 28(3): 187-194.

Yanes, E., Gil, K., Rebolledo, L., Rebolledo, A., Uriza, D., Martínez, O., Isidrón, M., Díaz, L., Lorenzo, J. C. y Simpson, J. (2012). Genetic diversity of Cuban pineapple germplasm assessed by AFLP markers. Crop Breed. Appl. Biotech. 12: 104-110. 